\title{
Insuficiência mitral: comparação entre o tratamento clínico e cirúrgico a médio prazo de acordo com a classe funcional
}

\author{
Kanim Kalil KASSAB* ${ }^{*}$ Amer Kalil KASSAB*
}

RBCCV 44205-581

\begin{abstract}
Kassab KK \& Kassab AK - Insuficiência mitral: comparação entre o tratamento clínico e cirúrgico a médio prazo de acordo com a classe funcional. Rev Bras Cir Cardiovasc 2002; 17(2): 20-23.

RESUMO: Casuística e Métodos: Analisamos, retrospectivamente, o índice de mortalidade a médio prazo de 113 doentes portadores de insuficiência mitral, diagnosticados clínica e ecocardiograficamente, e submetidos a tratamento clínico ou cirúrgico. A idade dos pacientes

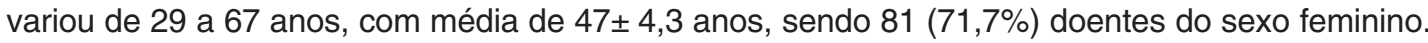
De acordo com a classificação de NYHA, 51 (45,1\%) doentes encontravam-se em classe funcional I, 24 (21,2\%) em classe II, 21 (18,6\%) em classe III e 17 (15,1\%) em classe IV.

Resultados: Após 4 anos, observamos que o taxa de mortalidade global foi de $21,2 \%$. Todos os $75(66,4 \%)$ doentes que se encontravam em classe funcional I e II receberam apenas tratamento clínico e apresentaram taxa de mortalidade de 12\%. Dos 38 pacientes que se encontravam em classe funcional III e IV, 18 (47,4\%) foram submetidos a tratamento cirúrgico e apresentaram taxa de mortalidade de $22,2 \%$, sendo $5,5 \%$ intra-hospitalar e $16,7 \%$ no período de 4 anos. Os 20 $(52,6 \%)$ doentes que recusaram a operação apresentavam condições clínicas semelhantes aos operados e foram submetidos a tratamento clínico, havendo índice de mortalidade de 55\%.

Conclusão: Concluímos que a insuficiência mitral apresenta alto índice de mortalidade e que o tratamento cirúrgico para os doentes que se encontram em classe funcional III e IV da NYHA apresenta menor índice de mortalidade a médio prazo quando comparado ao tratamento clínico, devendo ser indicado sempre quando possível.

DESCRITORES: Insuficiência da valva mitral, cirurgia. Insuficiência da valva mitral, terapia. Insuficiência da valva mitral, mortalidade. Valva mitral, cirurgia.
\end{abstract}

INTRODUÇÃO

A insuficiência mitral é considerada doença cardíaca comum, grave e de difícil controle, podendo o doente permanecer assintomático durante vários anos. $\mathrm{O}$ tratamento cirúrgico geralmente melhora a sintomatologia clínica, mas a disfunção ventricular residual, quando presente, apresenta prognóstico desfavorável a médio prazo.

A operação deve ser realizada precocemente tão logo o paciente comece a apresentar sintomatologia relacionada à disfunção ventricular esquerda.

O objetivo deste estudo é comparar o benefício a médio prazo do tratamento cirúrgico em relação ao tratamento clínico nos doentes portadores de insuficiência mitral e disfunção ventricular esquerda que se encontram em classe funcional III e IV da NYHA.

\section{CASUÍSTICA E MÉTODOS}

Foram incluídos em nosso estudo retrospectivo, através da análise de prontuários, 113 doentes portadores de insuficiência mitral, submetidos a tratamento clínico ou cirúrgico. Foram excluídos os portadores de insuficiência mitral secundária à rotura de músculo papilar, endocardite, insuficiência coronariana, doenças congênitas e trauma. A idade

Trabalho realizado no Serviço de Cirurgia Cardíaca - Pulmocor - Hospital Beneficência Portuguesa de São Paulo. São Paulo, SP, Brasil.

* Do Hospital Beneficência Portuguesa de São Paulo.

Endereço para correspondência: Dr. Kanim Kalil Kassab. Rua Vergueiro,1247 - apto.72 -Paraíso - São Paulo, SP, Brasil. CEP 01504-001. Tel. (11) 9302-3037. e.mail:kanim@ig.com.br 
dos pacientes variou de 29 a 67 anos (média de 47 \pm 4 ,3anos), sendo $81(71,7 \%)$ do sexo feminino.

O diagnóstico inicial foi feito pelo exame físico, complementado por ecocardiograma bidimensional transtorácico, que revelava regurgitação mitral que variava de + a $4+/ 6+$

O contato inicial com o doente e/ou familiares, para obtermos as informações necessárias para o desenvolvimento do estudo foi realizado via telefone. Os doentes foram divididos em três grupos: A, B e C.

Grupo A - classe funcional I e II: 75 doentes tratados clinicamente. doentes.

Grupo B e C - classe funcional III e IV: 38

Grupo B - 20 doentes não operados.

Grupo C - 18 doentes operados.

Todos os doentes foram operados por esternotomia mediana, seguida por pericardiotomia. Após heparinização com $4 \mathrm{mg} / \mathrm{kg}$ de peso eram canuladas a aorta ascendente e as 2 veias cava. Após a instalação de CEC, todos eram submetidos a hipotermia moderada $\left(28^{\circ} \mathrm{C}\right)$, pinçamento total e contínuo da aorta e proteção miocárdica com cardioplegia tipo St.Thomas a $4^{\circ} \mathrm{C}$ e atriotomia esquerda. Em todos os pacientes submetidos à troca valvar mitral preservamos a cúspide posterior, e naqueles submetidos à plastia valvar mitral foram preservadas ambas as cúspides.

Foi obtido o valor de odds ratio para a associação entre óbito e realização de operação, e respectivo intervalo de confiança $95 \%$. O teste do qui-quadrado foi realizado para verificação da significância estatística, estabelecendo-se como limite $p=0,05$.

\section{RESULTADOS}

Dos 113 doentes portadores de insuficiência mitral, $47(41,6 \%)$ apresentavam dispnéia e cansaço, 17 (15 $\%)$ queixa de palpitações, $31(27,4 \%)$ realizavam consulta de rotina devido à presença de sopro, 11 (9,7\%) queixa de precordialgia e $7(6,2 \%)$ com outras queixas.

O ecocardiograma bidimensional com doppler evidenciava acometimento de ambas as cúspides em 77 $(68,1 \%)$ doentes, da cúspide anterior em $26(23,1 \%)$ e da posterior em $10(8,8 \%)$; regurgitação mitral de leve a moderada em $30(26,5 \%)$ doentes e importante em 83 (73,4\%); fração de ejeção (FE) ${ }^{3} 60 \%$ em $32(28,3 \%)$ doentes e <60\% em 81(71.7\%); diâmetro atrial esquerdo £ 40mm em 26 (23\%) doentes e > $40 \mathrm{~mm}$ em $87(77 \%)$.

O eletrocardiograma evidenciava que $9(8,0 \%)$ doentes apresentavam fibrilação atrial, sendo que $1(0.9 \%)$ pertencia ao grupo A, $5(4.4 \%)$ ao B e $3(2.7 \%)$ ao C.

A cineangiocoronariografia com ventriculografia foi realizada em $81(71.7 \%)$ doentes, onde 7 (6,2\%) apresentavam coronariopatia associada. A hipertensão arterial estava presente em 38 (33,6 \%) e a fibrilação atrial em 21 (18,6 \%).

De acordo com a classificação de NYHA, 51 doentes $(45,1 \%)$ encontravam-se em classe I, 24 $(21,2 \%)$ em classe II, 21 (18,6\%) em classe III e 17 $(15,1 \%)$ em classe IV.

Os $75(66,4 \%)$ doentes que se encontravam em classe funcional I e II da NYHA foram tratados clinicamente e apresentaram taxa de mortalidade, após 4 anos, de $12 \%$.

Dos $38(33,6 \%)$ doentes que se encontravam em classe funcional III e IV da NYHA, $20(52,6 \%)$ foram tratados apenas clinicamente e $18(47,4 \%)$ foram submetidos a tratamento cirúrgico.

O risco de ir a óbito, em 4 anos, para os pacientes não operados foi 4 vezes maior do que para os submetidos a cirurgia (OR= 4,28; IC95\%), apresentando respectivamente índice de mortalidade de $55 \%$ e $22,2 \%(p=0,083)$.

Os pacientes submetidos ao tratamento cirúrgico apresentaram taxa de mortalidade intra-hospitalar de $5,5 \%$ e de $16,7 \%$ no período de 4 anos.

A plastia valvar foi realizada em $4(22,2 \%)$ doentes, não havendo óbitos, e o implante de prótese em 14 (77,8\%), sendo 11 biológicas e 3 mecânicas.

O taxa de mortalidade global após 4 anos foi de $21,2 \%$ e as causas de óbitos foram: insuficiência cardíaca congestiva (ICC) em $10(41,6 \%)$ doentes, acidente vascular cerebral hemorrágico $(\mathrm{AVCH})$ em 1 $(4.2 \%)$, acidente vascular cerebral isquêmico (AVCl) em $3(12,5 \%)$, endocardite infecciosa (EI) em 2 (8,3\%), insuficiência renal(IR) em 1 (4,2\%), arritmia (ARRIT) em $3(12,5 \%)$ e outras em $4(16,7 \%)$. As causas de óbitos estão distribuídas de acordo com a classe funcional da NYHA - Tabela 1.

\section{COMENTÁRIOS}

A maioria dos pacientes portadores de insuficiência mitral que procura auxílio médico apresenta como queixa principal dispnéia e cansaço, sendo que $66,3 \%$ encontrava-se em classe funcional I e II da NYHA. A incidência desta doença é maior no sexo feminino $(71,7 \%)$, acometendo prioritariamente ambas as cúspides, seguido da anterior e finalmente da posterior.

Sabemos que a taxa de mortalidade dos doentes portadores de insuficiência mitral, que se encontram em classe funcional I e II da NYHA após 5 e 10 anos é, respectivamente, de $18 \pm 4 \%$ e $33 \pm 9 \%$ e para aqueles que se encontram em classe funcional III e IV é de $86 \pm 9 \%$ em 5 anos, sendo que menos de 5 $\%$ chegam ao $10^{\circ}$ ano ${ }^{(1)}$.

Apesar da fração de ejeção não representar 
TABELA 1. DISTRIBUIÇÃO DAS CAUSAS DE ÓBITOS DE ACORDO COM A CLASSE FUNCIONAL NA INSUFICIÊNCIA MITRAL

\begin{tabular}{lcllclll}
\hline & ICC & AVCH & AVCI & EI & IR & ARRIT & OUTROS \\
GRUPO A & $1(4,2 \%)$ & $1(4,2 \%)$ & $2(8,3 \%)$ & 0 & 0 & $2(8,3 \%)$ & $3(12,5 \%)$ \\
GRUPO B & $7(29,1 \%)$ & 0 & $1(4,2 \%)$ & $1(4,2 \%)$ & $1(4,2 \%)$ & 0 & $1(4,2 \%)$ \\
GRUPO C & $2(8,3 \%)$ & 0 & 0 & $1(4,2 \%)$ & 0 & $1(4,2 \%)$ & 0 \\
TOTAL & $10(41,6 \%)$ & $1(4,2 \%)$ & $3(12,5 \%)$ & $2(8,4 \%)$ & $1(4,2 \%)$ & $3(12,5 \%)$ & $4(16,7 \%)$ \\
\hline
\end{tabular}

*Insuficiência cardíaca congestiva (ICC), Acidente vascular cerebral hemorrágico (AVCH), Acidente vascular cerebral isquêmico (AVCI), Endocardite infecciosa (EI), Insuficiência renal (IR), Arritmia (ARRIT).

parâmetro fidedigno para avaliar o grau de disfunção ventricular esquerda, já que estes doentes cursam inicialmente com elevação desta, alguns estudos citam que os doentes com $\mathrm{FE}{ }^{3}$ 60\% apresentam taxa de mortalidade em 5 e 10 anos, respectivamente, de $24 \pm$ $4 \%$ e $39 \pm 8 \%$ e os que apresentam FE < $60 \%$, respectivamente, de $47 \pm 11 \%$ e $60 \pm 12 \%$ (2-5).

Quando o diâmetro atrial esquerdo for $£ 40 \mathrm{~mm}$, os doentes apresentam taxa de mortalidade, respectivamente, em 5 e 10 anos, de $18 \pm 5 \%$ e $59 \pm 12 \%$ e quando > 40mm, respectivamente, de $47 \pm 9 \%$ e $75 \pm$ $10 \%{ }^{(6,7)}$.

É sabido que a fibrilação atrial é responsável por um grande número de complicações principalmente o tromboembolismo sistêmico e que o uso de anticoagulantes diminui a incidência desta. Em nossa casuística todos os doentes com fibrilação atrial faziam uso de anticoagulantes.

Observamos que $2(10 \%)$ doentes pertencentes ao grupo B e $1(5.6 \%)$ ao grupo $C$ apresentaram quadro de acidente vascular cerebral isquêmico (AVCl). Não temos número suficiente de casos para comparar estatisticamente a incidência de tromboembolismo sistêmico entre os grupos de doentes pertencentes a classe funcional III e IV que receberam tratamento clínico ou cirúrgico.

Analisando os dados obtidos anteriormente, observamos que a taxa de mortalidade a médio prazo é maior naqueles pacientes que apresentam fração de ejeção $£$ que $60 \%$ e diâmetro atrial esquerdo (3) que $40 \mathrm{~mm}$. A classificação funcional de NYHA, a fração de ejeção e o tamanho do átrio esquerdo podem ser usados como fatores prognósticos em relação à sobrevida a médio prazo destes doentes. Sendo assim, o ecocardiograma continua sendo o exame de eleição para determinar os parâmetros acima citados ${ }^{(8)}$.

Em nosso estudo, a maioria dos pacientes encontrava-se em classe funcional I e II da NYHA e quando tratados clinicamente apresentou taxa de mortalidade após quatro anos de $12 \%$. Aqueles que se encontravam em classe funcional III e IV da NYHA, que foram tratados cirurgicamente, apresentaram taxa de letalidade global de $22,2 \%$ após quatro anos, e quando tratados apenas clinicamente este taxa subia para $55 \%$. Evidencia-se um risco maior de óbito nos pacientes não operados. Apesar de não se verificar significância estatística, observou-se uma diferença marcante entre os grupos que poderá ser confirmada com a continuidade do estudo, envolvendo um número maior de pacientes.

Segundo a literatura ${ }^{(10-13)}$, a plastia mitral é considerada uma boa opção, devido à possibilidade de preservar as cúspides nativas da valva e o aparelho subvalvar, mantendo a geometria ventricular esquerda e evitando o implante de prótese e suas complicações.

Em nossa casuística houve apenas $4(22,2 \%)$ doentes submetidos à plastia mitral com preservação de ambas as cúspides e manutenção da função ventricular, número este insuficiente para uma análise estatística. Baseando-se neste pequeno número, acreditamos que há uma tendência dos doentes submetidos a esta operação apresentarem melhor evolução a médio prazo, quando comparados àqueles submetidos à troca valvar.

\section{CONCLUSÕES}

Em nosso estudo concluímos que :

- Os pacientes que se encontram no grupo I e II da NYHA podem ser tratados clinicamente com boa resposta;

- Nos pacientes que se encontram em classe funcional III ou IV, o tratamento cirúrgico apresenta melhor resposta e menor taxa de letalidade a médio prazo em relação aqueles no mesmo grupo tratados apenas clinicamente. 
RBCCV 44205-581

Kassab KK \& Kassab AK - Mitral regurgitation: comparison among clinical and surgical treatment medium term in agreement with the functional class. Rev Bras Cir Cardiovasc 2002; 17(2): 19-22.

ABSTRACT: Material and Methods: We analyzed retrospectively the medium term mortality index in 113 patients with mitral regurgitation diagnosed clinically and echocardiographic who have undergone clinical or surgical treatment since January 1995 until December 1998. The age ranged from 29 to 67 years (mean $47 \pm 4.3$ years ) and $81(71.7 \%)$ patients were female. In NYHA classification, $51(45.1 \%)$ patients belonging to functional class I, $24(21.3 \%)$ class II $21(18.6 \%)$ class III and 17 (15\%) class IV.

Results: After 4 years we observed that the mortality rate was $21.2 \%$. All the $75(66.4 \%)$ patients belonging to FC (NYHA) I and II, who received only clinical treatment presented mortality rate of $12 \%$. From the 38 patients belonging to FC (NYHA) III and IV, $18(47.4 \%)$ were submitted to surgical treatment and showed mortality rate of $22.2 \%$ : (5.5\% surgical mortality and $16.7 \%$ during 4 years). The $20(52.6 \%)$ patients that refused the surgery and presented similar clinical conditions to those operated, were treated clinically and demonstrated mortality rate of $55 \%$.

Conclusion: We conclude that the mitral regurgitation exhibit high mortality rate and that surgical treatment to the patients belonging to FC ( NYHA) III and IV reveals low mortality rate medium term when compared with clinical treatment.

DESCRIPTORS: Mitral valve insufficiency, surgery. Mitral valve insufficiency, therapy. Mitral valve insufficiency, mortality. Mitral valve, surgery.

REFERÊNCIAS BIBLIOGRÁFICAS

1- Phillips HR, Levine FH, Carter JE et al. - Mitral valve replacement for isolated mitral regurgitation: analysis of clinical course and late postoperative left ventricular ejection fraction. Am J Cardiol 1981; 48: 647-54.

2- Hochreiter C, Niles N, Devereux RB, Kligfield $\mathrm{P}$, Borer JS - Mitral regurgitation: relationship of noninvasive descriptors of right and left ventricular performance to clinical and hemodynamic findings and to prognosis in medically and surgically treated patients. Circulation 1986; 73:900-12.

3- Rich S, Sheikh A, Gallastegui J, Kondos GT, Mason T, Lam W - Determination of left ventricular ejection fraction by visual estimation during real-time two-dimensional echocardiography. Am Heart J 1982; 104: 603-6.

4- Quinones MA, Waggoner AD, Reduto LA et al. - A new, simplified and accurate method for determining ejection fraction with two-dimensional echocardiography. Circulation 1981; 64:744-53.

5- Vaziri SM, Larson MG, Benjamin EJ, Levy D Echocardiographic predictors of nonrheumatic atrial fibrilation: the Framingham Heart Study. Circulation 1994; 89:724-30.

6- Gehl LG, Mintz GK, Kotler MN, Segal BL - Left atrial volume overload in mitral regurgitation: a two dimensional echocardiografic study. Am J Cardiol 1982, 49: 33-8.
7- Burwash IG, Blackmore GL, Koilpillai CJ Usefulness of left atrial and left ventricular chamber sizes as predictors of the severity of mitral regurgitation. Am J Cardiol 1992; 70: 774-9.

8- Mintz GS, Kotler MN, Segal BL, Parry WR Two-dimensional echocardiographic recognition of rupture chordae tendineae. Circulation 1978; 57:24450.

9- Rosen SE, Borer JS, Hochreiter C et al. - Natural history of the asymptomatic/minimally symptomaitc patient with severe mitral regurgitation secondary to mitral valve prolapse and normal right and left ventricular performance. Am J Cardiol 1994; 74:374-80.

10-Luxereau P, Dorent R, De Gevigney G, Bruneval P, Chomette G, Delahaye G - Aetiology of surgically treated mitral regurgitation. Eur Heart J 1991; 12(Suppl.B):2-4.

11-Brandão CMA, Pomerantzeff PMA, Grinberg M, Stolf NAG, Jatene AD - Análise tardia de 291 pacientes submetidos à substituição valvar por próteses metálicas. Rev Bras Cir Cardiovasc 1995; 10: 50-5.

12-Braile DM, Volpe MA, Ramin SL, Souza DRS Tratamento cirúrgico das valvopatias - Parte 1. Rev Bras Cir Cardiovasc 1994; 9:113-22.

13-Braile DM, Volpe MA, Ramin SL, Souza DRS Tratamento cirúrgico das valvopatias - Parte 2. Rev Bras Cir Cardiovasc 1994; 9:159-68. 